\title{
RANDOM EFFECT BIVARIATE SURVIVAL MODELS AND STOCHASTIC COMPARISONS
}

\author{
RAMESH C. GUPTA, ${ }^{*}$ University of Maine \\ RAMESHWAR D. GUPTA, ${ }^{* *}$ University of New Brunswick
}

\begin{abstract}
In this paper we propose a general bivariate random effect model with special emphasis on frailty models and environmental effect models, and present some stochastic comparisons. The relationship between the conditional and the unconditional hazard gradients are derived and some examples are provided. We investigate how the well-known stochastic orderings between the distributions of two frailties translate into the orderings between the corresponding survival functions. These results are used to obtain the properties of the bivariate multiplicative model and the shared frailty model.
\end{abstract}

Keywords: Frailty model; environmental effect model; hazard gradient; bivarate multiplicative model; shared frailty model

2010 Mathematics Subject Classification: Primary 62N99; 62P99

Secondary 60E 15

\section{Introduction}

Random effect models have been widely used in the context of linear models. In linear models, the random effect is introduced as the error variable and the distribution of this error variable is reflected in the parameter estimates and other inferential problems. Besides this, the random effect models have been used in survival analysis to model the heterogeneity unexplained by the covariates. In assessing the effect of environmental factors, these models have been used as random environmental models. The dependence structure created by the random effects in survival analysis is quite different than in the linear models. Recently, Rizopoulos et al. (2008), using the random effect model, investigated the association structure between a longitudinal response process and the time to an event of interest using the shared parameter framework.

As explained above, random effect models are used in different disciplines. We will present our results in the context of survival analysis or, more specifically, in the context of frailty models where the frailty is modeled as an unobservable random effect. Clayton (1978) and Clayton and Cuzick (1985) introduced the proportional hazard frailty model, where a group of observations is assigned a random effect that acts multiplicatively on the baseline hazard function. The proportional hazard frailty model implies conditional independence-conditional on the frailty terms, the event times are independent. However, unconditionally, they are dependent.

Received 20 October 2009; revision received 11 February 2010.

* Postal address: Department of Mathematics and Statistics, University of Maine, Orono, ME 04469-5752, USA.

Email address: ramesh_gupta@umit.maine.edu

** Postal address: Department of Computer Science and Applied Statistics, University of New Brunswick, Saint John, E2L 4L5, Canada. 


\subsection{Frailty models}

As is well known, a particularly useful tool in handling heterogeneity unexplained by the observed covariates is the 'frailty model', introduced in Vaupel et al. (1979). The classical frailty model is given by

$$
\lambda(t \mid v)=v \lambda_{0}(t), \quad t>0,
$$

where $\lambda_{0}(t)$ is the baseline hazard independent of $v$.

Model (1.1) states that the hazard rate of an individual is the product of the individual specific quantity $v$ and the baseline hazard rate $\lambda_{0}(t)$ describing the age effect. We consider $v \lambda_{0}(t)$ to be the conditional failure rate of a random variable $T$ given $V=v$, where $V$ is the frailty or mixing random variable. Frailty models have often been used when groups of subjects have responses that are likely to be dependent in some general way. For example, in an animal carcinogeneity study, the responses of members of the same litter are not likely to be independent. Liang et al. (1995) discussed the use of frailty models when multiple events have been observed on the same subject.

It is well known that the choice of frailty distribution strongly affects the estimate of the baseline hazard as well as the conditional probabilities; see Hougaard (1984), (1991), (1995), (2000, pp. 213-262), Heckman and Singer (1984), and Agresti et al. (2004). Since different distributions of frailty give rise to different population level distributions of analyzing survival data, it is appropriate to investigate how the comparative effect of two frailties translates into the comparative effect on the resulting distributions. In the shared frailty models, the assumptions about the frailty distributions play an important role in the model's interpretation since the frailty distribution links the two processes of interest. For more discussion, see Rizopoulos et al. (2008) and Sargent (1998). In this connection, in the univariate case, Gupta and Kirmani (2006) investigated how well-known stochastic orderings between distributions of two frailties translate into orderings between the corresponding survival functions. More recently, in the univariate case, Gupta and Gupta (2009) studied a similar problem for a general frailty model which includes the classical frailty model (1.1) as well as the additive frailty model. It may be mentioned that, in the univariate setup, the above two papers reached results similar to the ones contained in the present paper.

For model (1.1), the population level hazard function is given by

$$
\lambda(t)=-\frac{\mathrm{d}}{\mathrm{d} t} \ln \bar{F}(t)
$$

where

$$
\bar{F}(t)=\mathrm{P}(T>t)=M_{V}\left(-\Lambda_{0}(t)\right),
$$

$M_{V}(\cdot)$ denotes the moment generating function of $V$, and $\Lambda_{0}(t)=\int_{0}^{t} \lambda_{0}(x) \mathrm{d} x$.

The overall population hazard function $\lambda(t)$ is related to the baseline hazard function $\lambda_{0}(t)$ by the relation

$$
\lambda(t)=\lambda_{0}(t) \mathrm{E}(V \mid T>t)
$$

Since

$$
\frac{\mathrm{d}}{\mathrm{d} t} \mathrm{E}(V \mid T>t)=-\lambda_{0}(t) \operatorname{var}(V \mid T>t)
$$

(see Gupta and Gupta (1996)), $\lambda(t) / \lambda_{0}(t)$ is a decreasing function of $t$. It can be seen that if $\mathrm{E}(V) \leq 1$ then $\lambda(t) \leq \lambda_{0}(t), t>0$, or, equivalently, $\bar{G}(t) / \bar{F}(t)$ is decreasing on $[0, \infty)$, where $\bar{G}(t)$ is the baseline survival function. In order to avoid identifiability problems, it is generally assumed that $\mathrm{E}(V)=1$. In the case of gamma frailty, see Hougaard (2000, p. 233). 
In this paper we study a general bivariate frailty model and present some stochastic comparisons using different distributions of the frailty. To this end, we define the bivariate hazard functions as follows.

Let $T_{1}$ and $T_{2}$ be two dependent random variables having absolutely continuous bivariate survival function $\bar{F}\left(t_{1}, t_{2}\right)=\mathrm{P}\left(T_{1}>t_{1}, T_{2}>t_{2}\right)$. Then the hazard (failure) rates, defined as

$$
\lambda^{(i)}\left(t_{1}, t_{2}\right)=-\frac{\partial}{\partial t_{i}} \ln \bar{F}\left(t_{1}, t_{2}\right), \quad i=1,2,
$$

are often used in demography, survival analysis, and biostatistics when analyzing bivariate survival data. Clearly, $\lambda^{(1)}\left(t_{1}, t_{2}\right)$ is the hazard rate of $T_{1}$ given $T_{2}>t_{2}$. Likewise, $\lambda^{(2)}\left(t_{1}, t_{2}\right)$ is the hazard rate of $T_{2}$ given $T_{1}>t_{1}$. The vector $\left(\lambda^{(1)}\left(t_{1}, t_{2}\right), \lambda^{(2)}\left(t_{1}, t_{2}\right)\right)$ is called the hazard gradient. It is well known that the hazard gradient uniquely determines the survival function. Thus, we consider the general bivariate frailty model

$$
\lambda^{(i)}\left(t_{1}, t_{2} \mid v\right)=\lambda^{(i)}\left(t_{1}, t_{2}, v\right), \quad i=1,2,
$$

where $v$ is the frailty associated with an individual.

As mentioned earlier, our aim in this paper is to develop the properties of the general bivariate frailty model (1.2) and obtain some results for the stochastic comparisons using different frailty distributions. As a special case, we will obtain results for the classical bivariate frailty model and the shared frailty model. The organization of this paper is as follows. After giving the aims and objectives together with the necessary definitions and background, we describe the general bivariate frailty model along with some of the properties in Section 2. In Section 3, our main results lie in seeing how the well-known stochastic orderings between distributions of two frailties translate into the orderings between the corresponding survival functions. These results are used, in Section 4, to investigate the stochastic order properties of the multiplicative model and the shared frailty model. Finally, some conclusions and comments are given in Section 5 .

Before proceeding further, we present some definitions and background for various stochastic comparisons.

Definition. Let $X$ and $Y$ be nonnegative absolutely continuous random variables with density functions $f(x)$ and $g(x)$, and survival functions $\bar{F}(x)$ and $\bar{G}(x)$, respectively. Then $X$ and $Y$ can be defined as follows.

(i) $X$ is said to be smaller than $Y$ in the likelihood ratio ordering, written as $X \leq_{\operatorname{lr}} Y$, if $f(x) / g(x)$ is nonincreasing in $x$.

(ii) $X$ is said to be smaller than $Y$ in the failure (hazard) rate ordering, written as $X \leq_{\mathrm{fr}} Y$, if $r_{F}(x) \geq r_{G}(x)$ for all $x$, where $r_{F}(x)$ and $r_{G}(x)$ are the hazard rates of $X$ and $Y$, respectively. This means that $\bar{G}(x) / \bar{F}(x)$ increases in $x$.

(iii) $X$ is said to be smaller than $Y$ in the stochastic ordering, written as $X \leq_{\text {st }} Y$, if $\bar{F}(x) \leq$ $\bar{G}(x)$ for all $x$.

(iv) $X$ is said to be smaller than $Y$ in the mean residual life ordering, written as $X \leq{ }_{\operatorname{mrl}} Y$, if $\mu_{F}(x) \leq \mu_{G}(x)$ for all $x$. Deshpande et al. (1990) showed that $X \leq \mathrm{mrl} Y$ if and only if $\int_{x}^{\infty} \bar{F}(u) \mathrm{d} u / \int_{x}^{\infty} \bar{G}(u) \mathrm{d} u$ is decreasing in $x$.

(v) Let $X$ and $Y$ be $n$-dimensional random vectors with hazard gradients

$$
\left(\lambda_{X}^{(1)}(x), \lambda_{X}^{(2)}(x), \ldots, \lambda_{X}^{(n)}(x)\right) \quad \text { and } \quad\left(\lambda_{Y}^{(1)}(y), \lambda_{Y}^{(2)}(y), \ldots, \lambda_{Y}^{(n)}(y)\right),
$$


respectively. Then $X \leq_{\text {whr }} Y$ (weak hazard rate order) if $\lambda_{X}^{(i)}(t) \geq \lambda_{Y}^{(i)}(t), i=1,2, \ldots, n$ and $t \in \mathbb{R}^{n}$; see Shaked and Shanthikumar (2007, p. 271). Note that this condition is weaker than the hazard rate orderings of vectors.

The following relations are well known:

$$
\begin{aligned}
& X \leq_{\mathrm{lr}} Y \quad \Longrightarrow \quad X \leq_{\mathrm{fr}} Y \quad \Longrightarrow \quad X \leq_{\mathrm{mrl}} Y \\
& \Downarrow \\
& X \leq_{\text {st }} Y \text {; }
\end{aligned}
$$

see Shaked and Shanthikumar (2007, p. 271).

\section{General bivariate frailty model}

Consider a general bivariate frailty model defined by the absolutely continuous joint survival function $\bar{F}\left(t_{1}, t_{2} \mid v\right)$, of a two-unit system, where $v$ is the frailty effect associated with the two variables. Define

$$
\begin{aligned}
\lambda^{(i)}\left(t_{1}, t_{2} \mid v\right) & =-\frac{\partial}{\partial t_{i}} \ln \bar{F}\left(t_{1}, t_{2} \mid v\right) \\
& =-\frac{\partial}{\partial t_{i}} \ln \bar{F}\left(t_{i} \mid T_{j}>t_{j}, v\right) \\
& =\frac{f\left(t_{i} \mid T_{j}>t_{j}, v\right)}{\bar{F}\left(t_{i} \mid T_{j}>t_{j}, v\right)}, \quad i, j=1,2, i \neq j .
\end{aligned}
$$

That is, $\lambda^{(i)}\left(t_{1}, t_{2} \mid v\right)$ is the failure rate function of the $i$ th unit with the $j$ th $(i \neq j)$ unit surviving until time $t_{j}$, conditional on the frailty variable $V$.

If $h(v)$ denotes the probability density function (PDF) of the random environmental effect $V$, then the unconditional joint PDF and the survival function are

$$
f\left(t_{1}, t_{2}\right)=\int_{0}^{\infty} f\left(t_{1}, t_{2} \mid v\right) h(v) \mathrm{d} v
$$

and

$$
\bar{F}\left(t_{1}, t_{2}\right)=\int_{0}^{\infty} \bar{F}\left(t_{1}, t_{2} \mid v\right) h(v) \mathrm{d} v,
$$

respectively. These functions are known as the population level joint density and survival functions. The population level failure rate functions are defined as

$$
\begin{aligned}
\lambda^{(i)}\left(t_{1}, t_{2}\right) & =-\frac{\partial}{\partial t_{i}} \ln \bar{F}\left(t_{1}, t_{2}\right) \\
& =-\frac{\partial}{\partial t_{i}} \ln \bar{F}\left(t_{i} \mid T_{j}>t_{j}\right) \\
& =\frac{f\left(t_{i} \mid T_{j}>t_{j}\right)}{\bar{F}\left(t_{i} \mid T_{j}>t_{j}\right)}, \quad i, j=1,2, i \neq j .
\end{aligned}
$$

In the following, we show that the population level hazard components are the averages of the conditional hazard components. 
Theorem 2.1. The population level failure rate function of the ith unit in a two-unit system with the $j$ th unit of fixed age $t_{j}$ is the expected value of $\lambda^{(i)}\left(t_{1}, t_{2} \mid v\right)$ with respect to the conditional distribution of the frailty effect $V$ given $T_{1}>t_{1}$ and $T_{2}>t_{2}$. That is,

$$
\lambda^{(i)}\left(t_{1}, t_{2}\right)=\mathrm{E}_{V \mid\left\{T_{1}>t_{1}, T_{2}>t_{2}\right\}}\left(\lambda^{(i)}\left(t_{1}, t_{2} \mid v\right)\right), \quad i=1,2 .
$$

It is assumed that the conditional distribution of $V$ given $T_{1}>t_{1}$ and $T_{2}>t_{2}$ is absolutely continuous, so that the conditional density exists.

Proof. We first show that

$$
h\left(v \mid T_{1}>t_{1}, T_{2}>t_{2}\right)=\frac{\bar{F}\left(t_{i} \mid T_{j}>t_{j}, v\right) h(v)}{\bar{F}\left(t_{i} \mid T_{j}>t_{j}\right)},
$$

where $h\left(v \mid T_{1}>t_{1}, T_{2}>t_{2}\right)$ is the conditional density of $V$ among survivors.

We have

$$
\begin{aligned}
h\left(v \mid T_{1}>t_{1}, T_{2}>t_{2}\right) & =\frac{-(\partial / \partial v) \mathrm{P}\left(V>v, T_{1}>t_{1}, T_{2}>t_{2}\right)}{\mathrm{P}\left(T_{1}>t_{1}, T_{2}>t_{2}\right)} \\
& =\frac{-(\partial / \partial v) \mathrm{P}\left(V>v, T_{i}>t_{i} \mid T_{j}>t_{j}\right)}{\mathrm{P}\left(T_{i}>t_{i} \mid T_{j}>t_{j}\right)} \\
& =\frac{-(\partial / \partial v) \int_{v}^{\infty} \int_{t_{i}}^{\infty} f\left(u, x_{i} \mid T_{j}>t_{j}\right) \mathrm{d} x_{i} \mathrm{~d} u}{\bar{F}\left(t_{i} \mid T_{j}>t_{j}\right)} \\
& =\frac{-(\partial / \partial v) \int_{v}^{\infty} \int_{t_{i}}^{\infty} f\left(x_{i} \mid T_{j}>t_{j}, u\right) h(u) \mathrm{d} x_{i} \mathrm{~d} u}{\bar{F}\left(t_{i} \mid T_{j}>t_{j}\right)} \\
& =\frac{-(\partial / \partial v) \int_{v}^{\infty} \bar{F}\left(x_{i} \mid T_{j}>t_{j}, u\right) h(u) \mathrm{d} u}{\bar{F}\left(t_{i} \mid T_{j}>t_{j}\right)} \\
& =\frac{\bar{F}\left(t_{i} \mid T_{j}>t_{j}, v\right) h(v)}{\bar{F}\left(t_{i} \mid T_{j}>t_{j}\right)}, \quad i, j=1,2, i \neq j .
\end{aligned}
$$

Therefore,

$$
\begin{aligned}
\lambda^{(i)}\left(t_{1}, t_{2}\right) & =\frac{f\left(t_{i} \mid T_{j}>t_{j}\right)}{\bar{F}\left(t_{i} \mid T_{j}>t_{j}\right)} \\
& =\frac{\int_{0}^{\infty} f\left(t_{i} \mid T_{j}>t_{j}, v\right) h(v) \mathrm{d} v}{\bar{F}\left(t_{i} \mid T_{j}>t_{j}\right)} \\
& =\int_{0}^{\infty} \lambda^{(i)}\left(t_{1}, t_{2} \mid v\right) \frac{\bar{F}\left(t_{i} \mid T_{j}>t_{j}, v\right) h(v) \mathrm{d} v}{\bar{F}\left(t_{i} \mid T_{j}>t_{j}\right)} \\
& =\int_{0}^{\infty} \lambda^{(i)}\left(t_{1}, t_{2} \mid v\right) h\left(v \mid T_{1}>t_{1}, T_{2}>t_{2}\right) \mathrm{d} v
\end{aligned}
$$

using (2.1). Thus, we have shown that

$$
\lambda^{(i)}\left(t_{1}, t_{2}\right)=\mathrm{E}_{V \mid\left\{T_{1}>t_{1}, T_{2}>t_{2}\right\}}\left(\lambda^{(i)}\left(t_{1}, t_{2} \mid v\right)\right), \quad i=1,2 .
$$

Under a very mild condition, the following result addresses the monotonicity of the distribution (survival) function of the random effect as a function of the ages of the two units. 
Theorem 2.2. If $\lambda^{(i)}\left(t_{1}, t_{2} \mid v\right)$ is an increasing function of $v, i=1,2$, then $H\left(v \mid T_{1}>\right.$ $\left.t_{1}, T_{2}>t_{2}\right)$ and $\bar{H}\left(v \mid T_{1}>t_{1}, T_{2}>t_{2}\right)$ are increasing and decreasing functions of $t_{i}$, respectively, where

$$
H\left(v \mid T_{1}>t_{1}, T_{2}>t_{2}\right)=\frac{\int_{0}^{v} \bar{F}\left(t_{i} \mid T_{j}>t_{j}, u\right) h(u) \mathrm{d} u}{\bar{F}\left(t_{i} \mid T_{j}>t_{j}\right)}, \quad i, j=1,2, i \neq j,
$$

is the conditional cumulative distribution function of the frailty variable among survivors and $\bar{H}=1-H$.

Proof. We have

$$
\begin{aligned}
\frac{\partial}{\partial t_{i}} H\left(v \mid T_{1}>t_{1}, T_{2}>t_{2}\right)= & \frac{\int_{0}^{v}\left(\partial / \partial t_{i}\right) \bar{F}\left(t_{i} \mid T_{j}>t_{j}, u\right) h(u) \mathrm{d} u}{\bar{F}\left(t_{i} \mid T_{j}>t_{j}\right)} \\
& -\frac{\left(\partial / \partial t_{i}\right) \bar{F}\left(t_{i}, T_{j}>t_{j}\right)}{\left[\bar{F}\left(t_{i}, T_{j}>t_{j}\right)\right]^{2}} \int_{0}^{v} \bar{F}\left(t_{i} \mid T_{j}>t_{j}, u\right) h(u) \mathrm{d} u \\
= & \frac{\int_{0}^{v} \bar{F}\left(t_{i} \mid T_{j}>t_{j}, u\right) h(u) \mathrm{d} u}{\bar{F}\left(t_{i} \mid T_{j}>t_{j}\right)}[-A(v)+A(\infty)],
\end{aligned}
$$

where

$$
A(v)=\frac{\int_{0}^{v} \lambda^{(i)}\left(t_{1}, t_{2} \mid u\right) \bar{F}\left(t_{i} \mid T_{j}>t_{j}, u\right) h(u) \mathrm{d} u}{\int_{0}^{v} \bar{F}\left(t_{i} \mid T_{j}>t_{j}, u\right) h(u) \mathrm{d} u} .
$$

We now show that $A(v)$ is increasing in $v>0$. We have

$$
\begin{aligned}
\frac{\mathrm{d}}{\mathrm{d} v} A(v)= & \frac{1}{\left(\int_{0}^{v} \bar{F}\left(t_{i} \mid T_{j}>t_{j}, u\right) h(u) \mathrm{d} u\right)^{2}} \\
& \times\left(\int_{0}^{v} \bar{F}\left(t_{i} \mid T_{j}>t_{j}, u\right) h(u) \mathrm{d} u\right) \lambda^{(i)}\left(t_{1}, t_{2} \mid v\right) \bar{F}\left(t_{i} \mid T_{j}>t_{j}, v\right) h(v) \\
& \left.\quad-\bar{F}\left(t_{i} \mid T_{j}>t_{j}, v\right) h(v) \int_{0}^{v} \lambda^{(i)}\left(t_{1}, t_{2} \mid u\right) \bar{F}\left(t_{i} \mid T_{j}>t_{j}, u\right) h(u) \mathrm{d} u\right) \\
= & \frac{\bar{F}\left(t_{i} \mid T_{j}>t_{j}, v\right) h(v)}{\left(\int_{0}^{v} \bar{F}\left(t_{i} \mid T_{j}>t_{j}, u\right) h(u) \mathrm{d} u\right)^{2}} \\
& \times\left(\int_{0}^{v}\left\{\lambda^{(i)}\left(t_{1}, t_{2} \mid v\right)-\lambda^{(i)}\left(t_{1}, t_{2} \mid u\right)\right\} \bar{F}\left(t_{i} \mid T_{j}>t_{j}, u\right) h(u) \mathrm{d} u\right) \\
> & 0,
\end{aligned}
$$

since $\lambda^{(i)}\left(t_{1}, t_{2} \mid v\right)>\lambda^{(i)}\left(t_{1}, t_{2} \mid u\right)$ for all $u<v$.

Corollary 2.1. If $\lambda^{(i)}\left(t_{1}, t_{2} \mid v\right)$ is an increasing function of $v, i=1,2$, then $\mathrm{E}\left(V \mid T_{1}>\right.$ $\left.t_{1}, T_{2}>t_{2}\right)$ is decreasing in $t_{i}, i=1,2$.

Proof. The hypothesis implies that $\bar{H}\left(v \mid T_{1}>t_{1}, T_{2}>t_{2}\right)$ is decreasing in $t_{i}>0$. Thus,

$$
\mathrm{E}\left(V \mid T_{1}>t_{1}, T_{2}>t_{2}\right)=\int_{0}^{\infty} \bar{H}\left(v \mid T_{1}>t_{1}, T_{2}>t_{2}\right) \mathrm{d} v
$$

is decreasing in $t_{i}>0, i=1,2$. 
Remark 2.1. The statement of Corollary 2.1 is a precise statement of the heuristically obvious fact that the weaker units in the population fail earlier than the others, so the remaining units are more robust than the rest.

Remark 2.2. The condition $\lambda^{(i)}\left(t_{1}, t_{2} \mid v\right)$ is an increasing function of $v$ will be satisfied by all the examples considered in the later sections.

The following results compare the frailty distribution of two groups, one with $T_{i}>t_{i 1}$ and $T_{j}>t_{j}$, and the other with $T_{i}>t_{i 2}$ and $T_{j}>t_{j}$, where $t_{i 1}<t_{i 2}, i, j=1,2, i \neq j$.

Theorem 2.3. If $\lambda^{(i)}\left(t_{1}, t_{2} \mid v\right)$ is an increasing function of $v$ then

$$
V\left|\left\{T_{i}>t_{i 2}, T_{j}>t_{j}\right\} \leq_{\operatorname{lr}} V\right|\left\{T_{i}>t_{i 1}, T_{j}>t_{j}\right\}, \quad 0<t_{i 1}<t_{i 2}, i=1,2 .
$$

Proof. We have

$$
\begin{aligned}
\frac{h\left(v \mid T_{i}>t_{i 2}, T_{j}>t_{j}\right)}{h\left(v \mid T_{i}>t_{i 1}, T_{j}>t_{j}\right)} & =\frac{\bar{F}\left(t_{i 2} \mid T_{j}>t_{j}, v\right) h(v)}{\bar{F}\left(t_{i 1} \mid T_{j}>t_{j}, v\right) h(v)} \frac{\bar{F}\left(t_{i 1} \mid T_{j}>t_{j}\right)}{\bar{F}\left(t_{i 2} \mid T_{j}>t_{j}\right)} \\
& =\exp \left\{-\int_{t_{i 1}}^{t_{i 2}} \lambda^{(i)}\left(t_{1}, t_{2}\right) \mathrm{d} t_{i}\right\} \frac{\bar{F}\left(t_{i 1} \mid T_{j}>t_{j}\right)}{\bar{F}\left(t_{i 2} \mid T_{j}>t_{j}\right)} .
\end{aligned}
$$

Since $\lambda^{(i)}\left(t_{1}, t_{2} \mid v\right)$ is an increasing function of $v, h\left(v \mid T_{i}>t_{i 2}, T_{j}>t_{j}\right) / h\left(v \mid T_{i}>\right.$ $\left.t_{i 1}, T_{j}>t_{j}\right)$ is decreasing in $v$. This means that the family of random variables $V \mid\left\{T_{i}>\right.$ $\left.t_{i}, T_{j}>t_{j}\right\}$ is decreasing in $t_{i}>0$ in the sense of the likelihood ratio.

We now present a general result comparing a random variable with its weighted version.

Lemma 2.1. Let $V_{1}$ and $V_{2}$ be two random variables with density functions $h_{1}(\cdot)$ and $h_{2}(\cdot)$ such that

$$
h_{2}(v)=\frac{g(v) h_{1}(v)}{\int_{0}^{\infty} g(v) h_{1}(v) \mathrm{d} v},
$$

where if $g(v)$ is a decreasing function of $v$ then $V_{1} \geq_{1 r} V_{2}$, and if $g(v)$ is an increasing function of $v$ then $V_{1} \leq \mathrm{lr} V_{2}$.

Proof. Since $h_{2}(v) / h_{1}(v)$ is nonincreasing in $v$, the result follows by definition.

The following result shows how the ordering between two frailties is preserved for surviving individuals.

Theorem 2.4. Let $V_{1}$ and $V_{2}$ be two frailty random variables such that $V_{2} \leq_{1 \mathrm{r}} V_{1}$. Then $V_{2}\left|\left\{T_{1}>t_{1}, T_{2}>t_{2}\right\} \leq \operatorname{lr} V_{1}\right|\left\{T_{1}>t_{1}, T_{2}>t_{2}\right\}$.

Proof. We have

$$
\begin{aligned}
H_{2}\left(v \mid T_{1}>t_{1}, T_{2}>t_{2}\right) & =\frac{\int_{0}^{v} \bar{F}\left(t_{1}, t_{2} \mid u\right) h_{2}(u) \mathrm{d} u}{\int_{0}^{\infty} \bar{F}\left(t_{1}, t_{2} \mid u\right) h_{2}(u) \mathrm{d} u} \\
& =\frac{\int_{0}^{v} \bar{F}\left(t_{1}, t_{2} \mid u\right) g(u) h_{1}(u) \mathrm{d} u}{\int_{0}^{\infty} \bar{F}\left(t_{1}, t_{2} \mid u\right) g(u) h_{1}(u) \mathrm{d} u} .
\end{aligned}
$$

This means that the distribution of $V_{2} \mid\left\{T_{1}>t_{1}, T_{2}>t_{2}\right\}$ is the weighted version of the distribution of $V_{1} \mid\left\{T_{1}>t_{1}, T_{2}>t_{2}\right\}$ by weight $g(v)$, a decreasing function of $v$. By applying Lemma 2.1 we obtain the result. 


\subsection{Examples}

Suppose that

$$
\bar{F}\left(t_{1}, t_{2} \mid v\right)=\mathrm{e}^{-v \psi\left(t_{1}, t_{2}\right)},
$$

where $\psi\left(t_{1}, t_{2}\right)$ is a differentiable function in both arguments such that $\bar{F}\left(t_{1}, t_{2} \mid v\right)$ is a conditional survival function.

Then

$$
\lambda^{(i)}\left(t_{1}, t_{2} \mid v\right)=v \frac{\partial}{\partial t_{i}} \psi\left(t_{1}, t_{2}\right), \quad i=1,2 .
$$

This gives (for $i=1,2$ )

$$
\begin{aligned}
\lambda^{(i)}\left(t_{1}, t_{2}\right) & =\mathrm{E}_{v \mid\left\{T_{1}>t_{1}, T_{2}>t_{2}\right\}}\left(\lambda^{(i)}\left(t_{1}, t_{2} \mid v\right)\right) \\
& =\int_{0}^{\infty} v \frac{\partial}{\partial t_{i}} \psi\left(t_{1}, t_{2}\right) h\left(v \mid T_{1}>t_{1}, T_{2}>t_{2}\right) \mathrm{d} v,
\end{aligned}
$$

where $h\left(v \mid T_{1}>t_{1}, T_{2}>t_{2}\right)$ is the PDF of $V$ given $T_{1}>t_{1}$ and $T_{2}>t_{2}$. Thus,

$$
\begin{aligned}
\lambda^{(i)}\left(t_{1}, t_{2}\right) & =\frac{\partial}{\partial t_{i}} \psi\left(t_{1}, t_{2}\right) \mathrm{E}\left(V \mid T_{1}>t_{1}, T_{2}>t_{2}\right) \\
& =\lambda_{0}^{(i)}\left(t_{1}, t_{2}\right) \mathrm{E}\left(V \mid T_{1}>t_{1}, T_{2}>t_{2}\right),
\end{aligned}
$$

where $\lambda_{0}^{(i)}\left(t_{1}, t_{2}\right)(i=1,2)$ is the $i$ th component of the hazard gradient without incorporating the frailty effect. Thus,

$$
\frac{\lambda^{(i)}\left(t_{1}, t_{2}\right)}{\lambda_{0}^{(i)}\left(t_{1}, t_{2}\right)}=\mathrm{E}\left(V \mid T_{1}>t_{1}, T_{2}>t_{2}\right)
$$

It can be verified that

$$
\frac{\partial}{\partial t_{i}} \mathrm{E}\left(V \mid T_{1}>t_{1}, T_{2}>t_{2}\right)=-\lambda_{0}^{(i)}\left(t_{1}, t_{2}\right) \operatorname{var}\left(V \mid T_{1}>t_{1}, T_{2}>t_{2}\right) .
$$

This means that $\lambda^{(i)}\left(t_{1}, t_{2}\right) / \lambda_{0}^{(i)}\left(t_{1}, t_{2}\right)$ is a decreasing function of $t_{i}, i=1,2$.

2.1.1. Special case. If $T_{1}$ and $T_{2}$ are conditionally independent given the frailty, then $\psi\left(t_{1}, t_{2}\right)=$ $A_{1}\left(t_{1}\right)+A_{2}\left(t_{2}\right), \lambda_{0}^{(1)}\left(t_{1}, t_{2}\right)=A_{1}^{\prime}\left(t_{1}\right)$, and $\lambda_{0}^{(2)}\left(t_{1}, t_{2}\right)=A_{2}^{\prime}\left(t_{2}\right)$, where the prime denotes the derivative.

The conditional survival function given $V=v$ is

$$
\bar{F}\left(t_{1}, t_{2} \mid v\right)=\exp \left\{-v\left(A_{1}\left(t_{1}\right)+A_{2}\left(t_{2}\right)\right)\right\} .
$$

The unconditional survival function is

$$
\bar{F}\left(t_{1}, t_{2}\right)=M_{V}\left[-\left(A_{1}\left(t_{1}\right)+A_{2}\left(t_{2}\right)\right)\right],
$$

where $M_{V}(\cdot)$ is the moment generating function of $V$.

In the following examples, we use this setup and assume that $T_{1}$ and $T_{2}$ are independent given the frailty variable. 


\subsubsection{Specific examples.}

Example 2.1. Suppose that $V$ has a gamma distribution with PDF

$$
h(v)=\frac{1}{\beta^{\alpha} \Gamma(\alpha)} v^{\alpha-1} \mathrm{e}^{-v / \beta}, \quad v>0, \alpha>0, \beta>0 .
$$

So

$$
\bar{F}\left(t_{1}, t_{2}\right)=\left[1+\beta A_{1}\left(t_{1}\right)+\beta A_{2}\left(t_{2}\right)\right]^{-\alpha} .
$$

The hazard components are given by

$$
\lambda^{(i)}\left(t_{1}, t_{2}\right)=-\frac{\partial}{\partial t_{i}} \ln \bar{F}\left(t_{1}, t_{2}\right)=\frac{\alpha \beta A_{i}^{\prime}\left(t_{i}\right)}{1+\beta A_{1}\left(t_{1}\right)+\beta A_{2}\left(t_{2}\right)}, \quad i=1,2 .
$$

Furthermore,

$$
\mathrm{E}\left(V \mid T_{1}>t_{1}, T_{2}>t_{2}\right)=\frac{\alpha \beta}{1+\beta A_{1}\left(t_{1}\right)+\beta A_{2}\left(t_{2}\right)}
$$

and

$$
\operatorname{var}\left(V \mid T_{1}>t_{1}, T_{2}>t_{2}\right)=\frac{\alpha \beta^{2}}{\left[1+\beta A_{1}\left(t_{1}\right)+\beta A_{2}\left(t_{2}\right)\right]^{2}} .
$$

The above expressions yield the square of the coefficient of variation of $V$ given $T_{1}>t_{1}$ and $T_{2}>t_{2}$ as $1 / \alpha$. It can be verified that a constant value of the coefficient of variation occurs only in the case of gamma frailty.

Example 2.2. Suppose that $V$ has an inverse Gaussian distribution with PDF

$$
h(v)=\left(2 \pi a v^{3}\right)^{-1 / 2} \exp \left\{-\frac{(b v-1)^{2}}{2 a v}\right\}, \quad v>0, a>0, b>0 .
$$

This gives

$$
\bar{F}\left(t_{1}, t_{2}\right)=\exp \left\{\frac{b}{a}\left(1-\left(1-\frac{2 a}{b^{2}}\left(A_{1}\left(t_{1}\right)+A_{2}\left(t_{2}\right)\right)\right)\right)^{-1 / 2}\right\} .
$$

The hazard components are given by

$$
\lambda^{(i)}\left(t_{1}, t_{2}\right)=\frac{A_{i}\left(t_{i}\right)}{\left[b^{2}+2 a\left(A_{1}\left(t_{1}\right)+A_{2}\left(t_{2}\right)\right)\right]^{1 / 2}}, \quad i=1,2 .
$$

Furthermore,

$$
\mathrm{E}\left(V \mid T_{1}>t_{1}, T_{2}>t_{2}\right)=\frac{1}{\left[b^{2}+2 a\left(A_{1}\left(t_{1}\right)+A_{2}\left(t_{2}\right)\right)\right]^{1 / 2}}
$$

and

$$
\operatorname{var}\left(V \mid T_{1}>t_{1}, T_{2}>t_{2}\right)=\frac{a}{\left[b^{2}+2 a\left(A_{1}\left(t_{1}\right)+A_{2}\left(t_{2}\right)\right)\right]^{3 / 2}}
$$




\section{Comparisons of frailty models}

There is no firm basis for choosing the probability distribution of the frailty random variable $V$. It is therefore important to see how the overall survival function of the $i$ th unit, $i=1,2$, responds to the change in the probability distribution of $V$. To be more precise, if the true model for the probability distribution of the frailty random variable is that of $V_{1}$ and the adopted model assumes the distribution of $V_{2}$, then we would like to know the relationship between the resulting random variables. Our main objective in this section is to see how some of the well-known stochastic orderings between $V_{1}$ and $V_{2}$ translate into the orderings between the component lifetimes.

Theorem 3.1. Let $\lambda^{(i)}\left(t_{1}, t_{2} \mid v\right)$ be an increasing function of $v>0, i=1,2$. If $V_{2} \leq \operatorname{lr} V_{1}$ then $\left(T_{11}, T_{21}\right) \leq_{\mathrm{whr}}\left(T_{12}, T_{22}\right)$.

Proof. From (2.1) we have

$$
\begin{aligned}
& \lambda_{v 1}^{(i)}\left(t_{1}, t_{2}\right)-\lambda_{v 2}^{(i)}\left(t_{1}, t_{2}\right) \\
&=\int_{0}^{\infty} \lambda^{(i)}\left(t_{1}, t_{2} \mid u\right) h_{1}\left(u \mid T_{1}>t_{1}, T_{2}>t_{2}\right) \mathrm{d} u \\
& \quad-\int_{0}^{\infty} \lambda^{(i)}\left(t_{1}, t_{2} \mid u\right) h_{2}\left(u \mid T_{1}>t_{1}, T_{2}>t_{2}\right) \mathrm{d} u \\
&=\lambda^{(i)}\left(t_{1}, t_{2} \mid u\right)\left[H_{1}\left(u \mid T_{1}>t_{1}, T_{2}>t_{2}\right)-H_{2}\left(u \mid T_{1}>t_{1}, T_{2}>t_{2}\right)\right]_{0}^{\infty} \\
&-\int_{0}^{\infty} \frac{\mathrm{d}}{\mathrm{d} u} \lambda^{(i)}\left(t_{1}, t_{2} \mid u\right)\left[H_{1}\left(u \mid T_{1}>t_{1}, T_{2}>t_{2}\right)-H_{2}\left(u \mid T_{1}>t_{1}, T_{2}>t_{2}\right)\right] \mathrm{d} u \\
&= \int_{0}^{\infty} \frac{\mathrm{d}}{\mathrm{d} u} \lambda^{(i)}\left(t_{1}, t_{2} \mid u\right)\left[H_{2}\left(u \mid T_{1}>t_{1}, T_{2}>t_{2}\right)-H_{1}\left(u \mid T_{1}>t_{1}, T_{2}>t_{2}\right)\right] \mathrm{d} u \\
&> 0 .
\end{aligned}
$$

The rest of the proof follows by using the fact that $\lambda^{(i)}\left(t_{1}, t_{2} \mid v\right)$ is an increasing function of $v$ and $V_{2} \leq_{\text {lr }} V_{1}$.

Before presenting the next result, we give the following definition and the composition formula.

Definition 3.1. A real-valued function $f(t, v)$ is said to be $\mathrm{RR}_{2}$ (reverse rule of order 2) or $\mathrm{TP}_{2}$ (total positive of order 2 ) on $[0, \infty) \times[0, \infty)$ if

$$
f\left(t_{1}, v_{1}\right) f\left(t_{2}, v_{2}\right) \leq f\left(t_{1}, v_{2}\right) f\left(t_{2}, v_{1}\right)
$$

or, respectively,

$$
f\left(t_{1}, v_{1}\right) f\left(t_{2}, v_{2}\right) \geq f\left(t_{1}, v_{2}\right) f\left(t_{2}, v_{1}\right)
$$

for all $0<t_{1}<t_{2}$ and $0<v_{1}<v_{2}$.

Remark 3.1. The concepts of $\mathrm{RR}_{2}$ and $\mathrm{TP}_{2}$ are used to study the dependence between two variables; see Karlin (1968, pp. 11-45).

The following statements are equivalent.

1. A real-valued function $f(t, v)$ is $\mathrm{RR}_{2}$ on $[0, \infty) \times[0, \infty)$. 
2. $f\left(t, v_{1}\right) / f\left(t, v_{2}\right)$ is increasing in $t>0$ and $0<v_{1}<v_{2}$.

3. $\partial^{2} \ln f(t, v) / \partial t \partial v<0$.

4. $f(t \mid v)$ or $f(v \mid t)$ is $\mathrm{RR}_{2}$, where $f(t \mid v)$ and $f(v \mid t)$ are the conditional densities.

Similarly, the following statements are also equivalent.

1. A real-valued function $f(t, v)$ is $\mathrm{TP}_{2}$ on $[0, \infty) \times[0, \infty)$.

2. $f\left(t, v_{1}\right) / f\left(t, v_{2}\right)$ is decreasing in $t>0$ and $0<v_{1}<v_{2}$.

3. $\partial^{2} \ln f(t, v) / \partial t \partial v>0$.

4. $f(t \mid v)$ or $f(v \mid t)$ is $\mathrm{TP}_{2}$, where $f(t \mid v)$ and $f(v \mid t)$ are the conditional densities.

See Holland and Wang (1987).

We now present the following result.

Theorem 3.2. Suppose that the conditional joint PDF of $T_{i}$ and $V$ given $T_{j}>t_{j}, i, j=$ $1,2, i \neq j$, is $\mathrm{RR}_{2}$. Then

(a) $V$ is stochastically decreasing in the right tail with respect to $T_{i}$, that is, $\bar{H}\left(v \mid T_{i}>\right.$ $t_{i}, T_{j}>t_{j}, i \neq j$ ) is a decreasing function of $t_{i}, i=1,2$;

(b) $T_{i}$ is stochastically decreasing in the right tail with respect to $V$, that is, $\bar{F}\left(t_{i} \mid V>\right.$ $v, T_{j}>t_{j}, i \neq j$ ) is a decreasing function of $v$.

Proof. See Shaked (1977).

We now present the following well-known composition formula, which will be used in the sequel.

Definition. (Composition formula.) Let $f(t, v)$ be an $\mathrm{RR}_{2}$ or $\mathrm{TP}_{2}$ function in $t \in \mathrm{R}$ and $v \in A$, and let $h_{i}(v)$ be a $\mathrm{TP}_{2}$ function on $\{1,2\} \times A$, where $h_{i}(v)$ is a probability density function in $v$ for each $i$. Then

$$
\Phi_{i}(t)=\int_{A} f(t, v) h_{i}(v) \mathrm{d} v
$$

is $\mathrm{RR}_{2}$ or, respectively, $\mathrm{TP}_{2}$ on $\{1,2\} \times \mathbb{R}$. For a proof, see Karlin (1968).

The following result shows how the likelihood ratio ordering of $V_{1}$ and $V_{2}$ is inherited by $T_{1}$ and $T_{2}$.

Theorem 3.3. Suppose that $V_{1} \leq \mathrm{lr} V_{2}$. If $f\left(t_{i} \mid v, T_{j}>t_{j}\right)$ is $\mathrm{RR}_{2}$ or $\mathrm{TP}_{2}$ on $[0, \infty) \times[0, \infty)$, then

$T_{i, v_{1}}\left|T_{j}>t_{j} \geq_{\operatorname{lr}} T_{i, v_{2}}\right| T_{j}>t_{j} \quad$ or, respectively, $\quad T_{i, v_{1}}\left|T_{j}>t_{j} \leq_{\operatorname{lr}} T_{i, v_{2}}\right| T_{j}>t_{j}$.

Proof. The condition $V_{1} \leq \mathrm{lr} V_{2}$ implies that $h_{2}(v) / h_{1}(v)$ is increasing in $v>0$. That is, the map $(k, v) \rightarrow h_{k}(v)$ is $\mathrm{TP}_{2}$ on $\{1,2\} \times[0, \infty)$. If $f\left(t_{i} \mid v, T_{j}>t_{j}\right)$ is $\mathrm{RR}_{2}$ or $\mathrm{TP}_{2}$ in $t_{i}$ and $v$ then, using the composition formula, the map $\left(k, t_{i}\right) \rightarrow f_{k}\left(t_{i} \mid T_{j}>t_{j}\right)$ is $\mathrm{RR}_{2}$ or, respectively, $\mathrm{TP}_{2}$ on $\{1,2\} \times[0, \infty)$, where $f_{k}\left(t_{i} \mid T_{j}>t_{j}\right)$ is given by

$$
f_{k}\left(t_{i} \mid T_{j}>t_{j}\right)=\int_{0}^{\infty} f\left(t_{i} \mid u, T_{j}>t_{j}\right) h_{k}(u) \mathrm{d} u, \quad k=1,2 .
$$

This implies that $f_{2}\left(t_{i} \mid T_{j}>t_{j}\right) / f_{1}\left(t_{i} \mid T_{j}>t_{j}\right)$ is decreasing or, respectively, increasing 
in $t_{i}>0$. Hence, $T_{i, v_{1}}\left|T_{j}>t_{j} \geq_{\operatorname{lr}} T_{i, v_{2}}\right| T_{j}>t_{j}$ or, respectively, $T_{i, v_{1}} \mid T_{j}>t_{j} \leq \operatorname{lr}$ $T_{i, v_{2}} \mid T_{j}>t_{j}$. This completes the proof.

The following result addresses the inheritance of failure rate orderings of $V_{1}$ and $V_{2}$ by $T_{1}$ and $T_{2}$.

\section{Theorem 3.4. Suppose that}

(a) $V_{1} \leq_{\text {fr }} V_{2}$; and

(b) $\lambda^{(i)}\left(t_{1}, t_{2} \mid v_{1}\right) \leq \lambda^{(i)}\left(t_{1}, t_{2} \mid v_{2}\right)$ or $\lambda^{(i)}\left(t_{1}, t_{2} \mid v_{1}\right) \geq \lambda^{(i)}\left(t_{1}, t_{2} \mid v_{2}\right), v_{1}<v_{2}$.

Then

$$
T_{i, v_{1}}\left|T_{j}>t_{j} \geq_{\text {fr }} T_{i, v_{2}}\right| T_{j}>t_{j}, \quad i, j=1,2, i \neq j,
$$

or, respectively, $\quad T_{i, v_{1}}\left|T_{j}>t_{j} \leq_{\mathrm{fr}} T_{i, v_{2}}\right| T_{j}>t_{j}, \quad i, j=1,2, i \neq j$.

Proof. Since $V_{1} \leq_{\mathrm{fr}} V_{2}, \bar{H}_{2}(v) / \bar{H}_{1}(v)$ is increasing in $v>0$, which is equivalent to the map $(k, v) \rightarrow \bar{H}_{k}(v)$ being $\mathrm{TP}_{2}$ on $\{1,2\} \times[0, \infty)$. Condition (b) implies that $\bar{F}\left(t_{i} \mid v, T_{j}>t_{j}\right)$ is $\mathrm{RR}_{2}$ or $\mathrm{TP}_{2}$ in $t_{i}$ and $v$ on $[0, \infty) \times[0, \infty)$. Also, (b) implies that

$$
\bar{F}\left(t_{i} \mid v_{1}, T_{j}>t_{j}\right) \geq \bar{F}\left(t_{i} \mid v_{2}, T_{j}>t_{j}\right)
$$

or, respectively,

$$
\bar{F}\left(t_{i} \mid v_{1}, T_{j}>t_{j}\right) \leq \bar{F}\left(t_{i} \mid v_{2}, T_{j}>t_{j}\right)
$$

for $v_{1}<v_{2}$. This means that $\bar{F}\left(t_{i} \mid v, T_{j}>t_{j}\right)$ is decreasing or, respectively, increasing in $v$. Define

$$
\bar{F}_{k}\left(t_{i} \mid T_{j}>t_{j}\right)=\int_{0}^{\infty} \bar{F}_{k}\left(t_{i} \mid u, T_{j}>t_{j}\right) h_{k}(u) \mathrm{d} u
$$

Then the map $\left(k, t_{i}\right) \rightarrow \bar{F}_{k}\left(t_{i} \mid T_{j}>t_{j}\right)$ is $\mathrm{RR}_{2}$ or, respectively, $\mathrm{TP}_{2}$ on $\{1,2\} \times[0, \infty)$. Therefore, $\bar{F}_{2}\left(t_{i} \mid T_{j}>t_{j}\right) / \bar{F}_{1}\left(t_{i} \mid T_{j}>t_{j}\right)$ is a decreasing or, respectively, increasing function of $t_{i}>0$. Hence, $\lambda_{v_{1}}^{(i)}\left(t_{1}, t_{2}\right) \leq \lambda_{v_{2}}^{(i)}\left(t_{1}, t_{2}\right)$ or, respectively, $\lambda_{v_{1}}^{(i)}\left(t_{1}, t_{2}\right) \geq \lambda_{v_{2}}^{(i)}\left(t_{1}, t_{2}\right)$ for all $t_{i}>0$. That is,

$$
T_{i, v_{1}}\left|T_{j}>t_{j} \geq_{\text {fr }} T_{i, v_{2}}\right| T_{j}>t_{j}
$$

or, respectively,

$$
T_{i, v_{1}}\left|T_{j}>t_{j} \leq_{\text {fr }} T_{i, v_{2}}\right| T_{j}>t_{j} .
$$

The following theorem shows the corresponding result for the stochastic orderings.

Theorem 3.5. If $V_{1} \leq_{\mathrm{st}} V_{2}$ and $\bar{F}\left(t_{i} \mid v, T_{j}>t_{j}\right)$ is a decreasing function of $v$, then $T_{i, v_{2}} \mid T_{j}>$ $t_{j} \leq_{\mathrm{st}} T_{i, v_{1}} \mid T_{j}>t_{j}, i, j=1,2, i \neq j$.

Proof. We have

$$
\begin{aligned}
\bar{F}_{2}\left(t_{i} \mid\right. & \left.T_{j}>t_{j}\right)-\bar{F}_{1}\left(t_{i} \mid T_{j}>t_{j}\right) \\
= & \int_{0}^{\infty} \bar{F}\left(t_{i} \mid v, T_{j}>t_{j}\right)\left[h_{2}(v)-h_{1}(v)\right] \mathrm{d} v \\
= & -\bar{F}\left(t_{i} \mid v, T_{j}>t_{j}\right)\left[\bar{H}_{2}(v)-\bar{H}_{1}(v)\right]_{0}^{\infty} \\
& +\int_{0}^{\infty} \frac{\mathrm{d}}{\mathrm{d} v} \bar{F}\left(t_{i} \mid v, T_{j}>t_{j}\right)\left[\bar{H}_{2}(v)-\bar{H}_{1}(v)\right] \mathrm{d} v \\
= & \int_{0}^{\infty} \frac{\mathrm{d}}{\mathrm{d} v} \bar{F}\left(t_{i} \mid v, T_{j}>t_{j}\right)\left[\bar{H}_{2}(v)-\bar{H}_{1}(v)\right] \mathrm{d} v .
\end{aligned}
$$


Since $\bar{F}\left(t_{i} \mid v, T_{j}>t_{j}\right)$ is a decreasing function of $v$ and $H_{2}(v) \geq H_{1}(v)$ for all $v>0$, we have $\bar{F}_{2}\left(t_{i} \mid T_{j}>t_{j}\right) \leq \bar{F}_{1}\left(t_{i} \mid T_{j}>t_{j}\right)$ for all $t_{i}>0$. That is, $\mathrm{T}_{i, v_{2}}\left|T_{j}>t_{j} \leq_{\mathrm{st}} T_{i, v_{1}}\right| T_{j}>t_{j}$.

\section{Multiplicative model}

We now consider the following model as a special case:

$$
\lambda^{(i)}\left(t_{1}, t_{2} \mid v\right)=v \lambda_{0}^{(i)}\left(t_{1}, t_{2}\right), \quad t_{1}>0, t_{2}>0, v>0,
$$

where $\lambda_{0}^{(i)}\left(t_{1}, t_{2}\right)$ is the baseline failure rate of the $i$ th unit without taking into account the frailty effect and is independent of $v$. In this case

$$
\bar{F}\left(t_{1}, t_{2} \mid v\right)=\left[\bar{G}\left(t_{1}, t_{2}\right)\right]^{v}, \quad v>0,
$$

where $\bar{G}\left(t_{1}, t_{2}\right)$ is the joint baseline survival function. This gives the unconditional survival function as

$$
\bar{F}\left(t_{1}, t_{2}\right)=\int_{0}^{\infty}\left[\bar{G}\left(t_{1}, t_{2}\right)\right]^{v} h(v) \mathrm{d} v, \quad v>0,
$$

where $h(v)$ is the PDF of the frailty effect $V$.

We now present the following result.

Theorem 4.1. For model (4.1), the following statements hold.

(a) The ith component of the population level failure rate is given by

$$
\lambda^{(i)}\left(t_{1}, t_{2}\right)=\lambda_{0}\left(t_{1}, t_{2}\right) \mathrm{E}_{V}\left(V \mid T_{1}>t_{1}, T_{2}>t_{2}\right)
$$

(b) $H\left(v \mid T_{1}>t_{1}, T_{2}>t_{2}\right)$ is an increasing function of $t_{i}, i=1,2$.

(c) $\mathrm{E}_{V}\left(V \mid T_{1}>t_{1}, T_{2}>t_{2}\right)$ is decreasing in $t_{i}>0, i=1,2$. Moreover, if $\lambda_{0}^{(i)}\left(t_{1}, t_{2}\right)$ is decreasing in $t_{i}>0$ then $\lambda^{(i)}\left(t_{1}, t_{2}\right)$ is decreasing in $t_{i}>0$. That is, $T_{i} \mid T_{j}>t_{j}, i, j=$ $1,2, i \neq j$, is of decreasing failure rate.

(d) $V\left|\left\{T_{i}>t_{i 2}, T_{j}>t_{j}\right\} \leq_{\operatorname{lr}} V\right|\left\{T_{i}>t_{i 1}, T_{j}>t_{j}\right\}$ for all $t_{i 1}<t_{i 2}, i, j=1,2, i \neq j$.

(e) If $V_{2} \leq_{\operatorname{lr}} V_{1}$ then $V_{2}\left|\left\{T_{1}>t_{1}, T_{2}>t_{2}\right\} \leq_{\operatorname{lr}} V_{1}\right|\left\{T_{1}>t_{1}, T_{2}>t_{2}\right\}$.

Proof. The proof of (a) follows from Theorem 2.1. For parts (b)-(e), since $\lambda^{(i)}\left(t_{1}, t_{2} \mid v\right)=$ $v \lambda_{0}^{(i)}\left(t_{1}, t_{2}\right)$ is an increasing function of $v$, Theorems 2.2-2.3 and Corollary 2.1 apply.

We now present the following result, which addresses the monotonicity of the conditional survival function of the $i$ th component given $V$ and the survival of the $j$ th component.

Theorem 4.2. The random variable $T_{i}$ is stochastically decreasing in the right tail with respect to $V$ given $T_{j}>t_{j}, i=1,2$. That is, $\bar{F}\left(t_{i} \mid v, T_{j}>t_{j}\right)$ is a decreasing function of $v>0$.

Proof. We have

$$
\begin{aligned}
& f\left(t_{i} \mid v, T_{j}>t_{j}\right)=\lambda^{(i)}\left(t_{1}, t_{2} \mid v\right) \bar{F}\left(t_{i} \mid v, T_{j}>t_{j}\right)=v \lambda_{0}^{(i)}\left(t_{1}, t_{2}\right)\left[\bar{G}\left(t_{i} \mid T_{j}>t_{j}\right)\right]^{v} . \\
& \text { If } 0<v_{1}<v_{2}<\infty \text { then }
\end{aligned}
$$

$$
\frac{f\left(t_{i} \mid v_{2}, T_{j}>t_{j}\right)}{f\left(t_{i} \mid v_{1}, T_{j}>t_{j}\right)}=\frac{v_{2}}{v_{1}}\left[\bar{G}\left(t_{i} \mid T_{j}>t_{j}\right)\right]^{v_{2}-v_{1}}
$$


is a decreasing function of $t_{i}>0$. Therefore, the conditional density of $T_{i}$ given $\left(v, T_{j}>t_{j}\right)$ is $\mathrm{RR}_{2}$ in $t_{i}$ and $v$ on $[0, \infty) \times[0, \infty)$. Hence, the joint PDF of $T_{i}$ and $V$ given $T_{j}>t_{j}$ is $\mathrm{RR}_{2}$. The result now follows from Theorem 3.2(b).

The following theorem shows how the stochastic comparisons between $V_{1}$ and $V_{2}$ translate into stochastic comparisons between $T_{1}$ and $T_{2}$.

Theorem 4.3. Let $A \in\{\mathrm{lr}, \mathrm{fr}, \mathrm{st}\}$. If $V_{1} \leq_{A} V_{2}$ then

$$
T_{i, v_{1}}\left|T_{j}>t_{j} \geq_{A} T_{i, v_{2}}\right| T_{j}>t_{j}, \quad i, j=1,2, i \neq j .
$$

Proof. (a) Since $f\left(t_{i} \mid v, T_{j} . t_{j}\right)$ is $\mathrm{RR}_{2}$ in $t_{i}$ and $v$, the result for lr follows from Theorem 3.4. (b) Since $\lambda^{(i)}\left(t_{1}, t_{2} \mid v\right)=v \lambda_{0}^{(i)}\left(t_{1}, t_{2}\right)$ is an increasing function of $v$, the result for fr follows from Theorem 3.4.

(c) Since $\bar{F}\left(t_{i} \mid v, T_{j}>t_{j}\right)=\left[\bar{G}\left(t_{i} \mid T_{j}>t_{j}\right)\right]^{v}$ is decreasing in $v>0$, the result for st follows from Theorem 3.5.

\subsection{Shared frailty model}

We now consider the following model, known as the shared frailty model:

$$
\lambda^{(i)}\left(t_{1}, t_{2} \mid v\right)=v \lambda_{0 i}\left(t_{i}\right), \quad i=1,2,
$$

where $\lambda_{0 i}\left(t_{i}\right)$ is the baseline failure rate of the $i$ th unit, independent of the other unit and of $v$. In this case

$$
\bar{F}\left(t_{1}, t_{2} \mid v\right)=\left[\bar{G}_{1}\left(t_{1}\right) \bar{G}_{2}\left(t_{2}\right)\right]^{v}, \quad v>0,
$$

where $\bar{G}_{i}\left(t_{i}\right)$ is the baseline survival function of the $i$ th unit. Thus,

$$
\bar{F}\left(t_{1}, t_{2}\right)=\int_{0}^{\infty}\left[\bar{G}_{1}\left(t_{1}\right) \bar{G}_{2}\left(t_{2}\right)\right]^{v} h(v) \mathrm{d} v,
$$

where $h(v)$ is the PDF of $V$.

Remark 4.1. It is easily seen that the shared frailty model is a special case of the multiplicative model discussed above, and all the results of Theorems 4.1-4.3 apply.

Remark 4.2. A more general, shared random effect model has been studied in Rizopoulos et al. (2008), who investigated the association structure between a longitudinal response and survival processes.

\section{Conclusion and comments}

In this paper we presented a general bivariate random effect model. Random effect models are used in various disciplines. For example, in survival analysis they are used as frailty models and in problems related to the environment they are used as environmental effect models. We presented our results in the context of frailty models and studied their properties. We also investigated the effect on the survival by using two different frailty distributions. The corresponding results for the multiplicative and shared frailty models were also derived. The two examples presented illustrate the effect on the survival by incorporating the frailty effect. It is hoped that our results will be useful to researchers dealing with random effect models. 


\section{Acknowledgements}

The authors are thankful to the anonymous referee for some useful comments which enhanced the presentation. Research of the second author was supported by a grant from the Natural Sciences and Engineering Council of Canada.

\section{References}

Agresti, A., Caffo, B. and Ohman-Strickland, P. (2004). Examples in which misspecification of a random effects distribution reduces efficiency, and possible remedies. Comput. Statist. Data Anal. 47, 639-653.

Clayton, D. G. (1978). A model for association in bivariate life tables and its application in epidemiological studies of familial tendency in chronic disease incidence. Biometrika 65, 141-151.

Clayton, D. and Cuzick, J. (1985). Multivariate generalizations of the proportional hazards model. J. R. Statist. Soc. A 148, 82-117.

Deshrande, J. V., Singh, H., Bagai, I. And JaIn, K. (1990). Some partial orders describing positive ageing. Commun. Statist. Stoch. Models 6, 471-481.

Gupta, P. L. ANd Gupta, R. C. (1996). Ageing characteristics of the Weibull mixtures. Prob. Eng. Inf. Sci. 10, 591-600.

Gupta, R. C. AND Gupta, R. D. (2009). General frailty model and stochastic orderings. J. Statist. Planning Infer. 139, 3277-3287.

Gupta, R. C. And Kirmani, S. N. U. A. (2006). Stochastic comparisons in frailty models. J. Statist. Planning Infer. 136, 3647-3658.

Heckman, J. And Singer, B. (1984). The identifiability of the proportional hazard model. Rev. Econom. Stud. 51, 231-241.

Holland, P. W. And Wang, Y. J. (1987). Dependence function for continuous bivariate densities. Commun. Statist. Theory Meth. 16, 863-876.

HougaARD, P. (1984). Life table methods for heterogeneous populations: distributions describing the heterogeneity. Biometrika 71, 75-83.

Hougaard, P. (1991). Modelling heterogeneity in survival data. J. Appl. Prob. 28, 695-701.

HougaArd, P. (1995). Frailty models for survival data. Lifetime Data Anal. 1, 255-273.

HougaArd, P. (2000). Analysis of Multivariate Survival Data. Springer, New York.

Karlin, S. (1968). Total Positivity, Vol. I. Stanford University Press.

Liang, K.-Y., Self, S. G., Bandeen-Roche, K. J. And Zeger, S. L. (1995). Some recent developments for regression analysis of multivariate failure time data. Lifetime Data Anal. 1, 403-415.

Rizopoulos, D., Verbeke, G. And Molenberghs, G. (2008). Shared parameter models under random effects misspecification. Biometrika 95, 63-74.

SARgENT, D. J. (1998). A general framework for random effects survival analysis in the Cox proportional hazards setting. Biometrics 54, 1486-1497.

ShaKed, M. (1977). A family of concepts of dependence for bivariate distributions. J. Amer. Statist. Assoc. 72, 642-650.

Shaked, M. And Shanthikumar, J. G. (2007). Stochastic Orders. Springer, New York.

Vaupel, J. W., Manton, K. G. and Stallard, E. (1979). The impact of heterogeneity in individual frailty on the dynamics of mortality. Demography 16, 439-454. 